\title{
PUBBLICAZIONI RECENTI.
}

6. Novi - Trattato di Algebra superiore. Parte I: Analisi Algebrica. - Firenze, 1863.

Paiwin - Propriétés des points d'inflexion des courbes du $3^{\text {e }}$ ordre et des points de rebroussement des courbes de la $3^{\mathrm{e}}$ classe (Extrail des Mémoires de la Société des sciences, de lagriculture et des arts de Lille).

G. SALMON - A treatise on conic sections etc. Fourth edition. - London, 1863.

Poudra - OEuvres des Desargues - Deux tomes. Paris, 1864.

JunghanN - Tetraedrometrie. 2 Th. Gotha 1862-63.

R. Townsend - Chapters on the modern Geometry of the point, line and circle etc. Vol. 1. - Dublin, 1863.

Marsano - Considerazioni sul triangolo rettilineo. - Genova, 1863.

H. Weissenborn - Die Element der Planimetrie. - Halle, 1864.

Praart - Essai d'une théorie géométrique des surfaces. - Paris, 1863.

Lucas - Études analytiques sur la théorie générale des courbes planes. - Paris, 1864.

Briot - Essais sur la théorie Mathématique de la lumière. - Paris, 1864.

J. De la Gournerie - Mémoire sur la surface engendrée par la révolution d'une conique autour d'une droite située d'une manière quelconque dans l'espace. - Paris, 1863.

L.JjEdne-Dirichlet - Vorlesungen über Zahlentheorie, heransgegeben von R. DedeKind - Braunschweig, 1863.

G. Salmon - Analytische Geometrie des Raumes, deutsch bearbeitet von W. Fredler. I Theil. Die Element und die Theorie der Flächen zweiten grades. - Leipzig, 1863,

E. Catalan - Mémoire en réponse à la question suivante: Trouver les lignes de courbure du lieu des points dont la somme des distances à deux droites qui se coupent est constante. - Bruxelles, 1864 .

J. Bertrand - Traité de Calcul différentiel et de Calcul intégral. - Calcul différentiel. - Paris, 1864.

L. Crenona - Nuove ricerche di geometria pura sulle cubiche gobbe ed in ispecie sulla parabola gobba. Bologna, 1863.

C. TAYLOR - Geometrical conies, including anharmonic ratio and projection. - London, 1863.

Plana Jean. Mémoire sur le mouvement du centre de gravité d'un corps solide lancé vers la terre entre les centres de la lune, et de la terre supposés finis immédiatement après l'impulsion. (Dalle Memorie dell' Accademia di Torino tom. XX. Serie secanda 1863 ).

- Réflexions sur la préface d'un Mémoire du Lagrange intitulé Solution d'un problème d'arithmétique publié dans le Tom. IV des Miscellanea Taurinensis. (Dalle Memorie etc.).

- Mémoire sur la théorie des nombres. (Dalle Memorie etc.).

- Réflexions sur les objections soulevées par Arag contre la priorité de Galilé pour la double decouverte des taches solaires noires, et de la rotation uniforme du globo du soleil. (Dalle Memorie etc.

- Mémoire sur la théorie des trascendantes elliptiques. (Dalle Memorie etc.).

- Note sur l'origine de la fraction $\mathrm{W}$ définie au commencement du premier $\$$ du Mémoire sur la théorie des trascendantes élliptiques. (Dalle Memorie etc.). 